\title{
A network pharmacology approach to explore Dahuang on colon cancer
}

\author{
Mai Wang ${ }^{1}$,Yinyue $\mathrm{Wang}^{1}$, and Shu Wang ${ }^{1, *}$ \\ ${ }^{1}$ Hebei North University,075000,Zhangiiakou,China
}

\begin{abstract}
To investigate the mechanism of action of Rhubarb (Dahuang) in the treatment of colon cancer by network pharmacological method. TCMSP database was used to screen the active components and action targets of Rhubarb, Genecards database was used to screen the relevant action targets of colon cancer, Venny 2.1.0 software was used to make the intersection of Rhubarb and colon cancer, and the potential targets of Rhubarb for the treatment of colon cancer were obtained.Cytoscape 3.7.2 software and the STRING database were used to construct the component-target network of Rhubarb active components and colon cancer related targets. The important targets were obtained by analyzing the network, and the GO and KEGG pathway enrichment analysis was performed on them using Metascape database. Sixty active ingredients including EUPATIN, Mutatochrome, Physciondiglucoside, Procyanidin B-5,3' -o-gallate, rhin were screened from Rhubarb, which could act on 61 colon cancer related targets.Among them, 13 targets, including PTGS2, IL1B and Bax, are important targets, which may be involved in various biological processes such as the response to the regulation of colon cancer nutritional level, the homeostasis of multicellular organisms and the regulation of peptide secretion through the regulation of PI3K and other pathways. Rhubarb can participate in the treatment of colon cancer through multi-target and multi-pathway.
\end{abstract}

\section{Introduction}

With the development of big data, network pharmacology has a profound influence on the prediction of the mechanism of Chinese herbal medicine against disease. As a traditional Chinese medicine, Rhubarb has many pharmacological effects. Colon cancer as one of the common cancers, in addition to the traditional chemotherapy has no other way of treatment. However, the harm of chemotherapy to human body can not be ignored. In this study, network pharmacological methods were used to explore the mechanism of Rhubarb in the treatment of colon cancer, revealing the complex molecular mechanism of Rhubarb in the treatment of colon cancer at the level of target and pathway, and providing new ideas and new directions for the study of the mechanism of action of Rhubarb in the treatment of colon cancer.

\section{Methods and results}

\subsection{Potential active components of Rhubarb and their action targets}

System in Chinese medicine pharmacological analysis platform (TCMSP, http://lsp.nwu.edu.cn/tcmsp.php) ${ }^{[1]}$ to retrieve the Rhubarb potential active ingredients, in accordance with the oral bioavailability (OB) $30 \%$ or higher, classes, medicinal standard screening (DL) of 0.18 or higher ${ }^{[2-3]}$. TCMSP database was used to search the action targets of the active ingredients obtained in the previous step, and the targets were mapped into standard gene names (gene IDs) through Uniprot database (https:// www.uniprot.org) to obtain the active ingredients and action targets of Rhubarb. A total of 16 active ingredients were screened out (Table 1).

Table1. Basic information of active ingredients in Rhubarb

\begin{tabular}{|c|c|c|c|}
\hline $\begin{array}{l}\text { TCMSP } \\
\text { NUMBER }\end{array}$ & INGREDIENTS & $\mathrm{OB} \%$ & DL $\%$ \\
\hline MOL002235 & EUPATIN & 50.8 & 0.41 \\
\hline MOL002251 & Mutatochrome & 48.64 & 0.61 \\
\hline MOL002259 & Physciondiglucoside & 41.65 & 0.63 \\
\hline MOL002260 & $\begin{array}{l}\text { Procyanidin B-5,3'- } \\
\text { O-gallate }\end{array}$ & 31.99 & 0.32 \\
\hline MOL002268 & rhein & 47.07 & 0.28 \\
\hline MOL002276 & Sennoside E_qt & 50.69 & 0.61 \\
\hline MOL002280 & $\begin{array}{l}\text { Torachrysone-8-O- } \\
\text { beta-D-(6'-oxayl)- } \\
\text { glucoside }\end{array}$ & 43.02 & 0.74 \\
\hline MOL002281 & Toralactone & 46.46 & 0.24 \\
\hline MOL002288 & $\begin{array}{l}\text { Emodin-1-O-beta- } \\
\text { D-glucopyranoside }\end{array}$ & 44.81 & 0.8 \\
\hline MOL002293 & Sennoside D_qt & 61.06 & 0.61 \\
\hline MOL002297 & Daucosterol_qt & 35.89 & 0.7 \\
\hline MOL002303 & palmidin A & 32.45 & 0.65 \\
\hline MOL000358 & beta-sitosterol & 36.91 & 0.75 \\
\hline MOL000471 & aloe-emodin & 83.38 & 0.24 \\
\hline
\end{tabular}

*Corresponding author:wangshu388@163.com 
gallic acid-3-O-(6'-

\begin{tabular}{llrr} 
MOL000554 & $\begin{array}{l}\text { O-galloyl)- } \\
\text { glucoside }\end{array}$ & 30.25 & 0.67 \\
MOL000096 & $\begin{array}{l}\text { (-)-catechin } \\
\text { gate }\end{array}$ & 49.68 & 0.24 \\
\hline
\end{tabular}

\subsection{Target of Rhubarb in the treatment of colon cancer}

Colon cancer related target genes were retrieved from GeneCards database (https://www.genecards.org) ${ }^{[4]}$. Then, the target gene of colon cancer was mapped to the target gene of Rhubarb active ingredient to screen, and the intersection was taken to obtain the potential target of Rhubarb in the treatment of colon cancer, and the Venny diagram was made. A total of 92 active component targets of Rhubarb and 1669 targets of colon cancer were retrieved, and 61 targets were obtained by mapping them together, which were potential target genes of Rhubarb for the treatment of colon cancer (Table 2, Fig.1).

Table2. Potential target genes of Rhubarb in treatment of colon cancer

\begin{tabular}{|c|c|c|c|c|c|}
\hline \multicolumn{6}{|c|}{ Name of gene target } \\
\hline $\begin{array}{l}\text { ADH1 } \\
\text { C }\end{array}$ & RRM1 & AR & F3 & $\begin{array}{l}\text { CDKN1 } \\
\text { A }\end{array}$ & EGF \\
\hline NR3C1 & $\begin{array}{l}\text { SLC16A } \\
7\end{array}$ & ESR2 & ARG1 & $\begin{array}{l}\text { TNFSF1 } \\
5\end{array}$ & MMP1 \\
\hline PTGS2 & MTR & PRSS1 & CASP3 & ТР63 & PPARG \\
\hline CTSD & IDH1 & KDR & $\begin{array}{l}\text { SLC6A } \\
4\end{array}$ & FASN & $\begin{array}{l}\text { CYP1A } \\
1\end{array}$ \\
\hline HDC & TF & ESR1 & BCL2 & PCNA & CSF2 \\
\hline PTGS1 & GPT & MMP9 & BAX & MYC & ACTA2 \\
\hline BCHE & SDHA & RXRA & CASP9 & IL1B & BTK \\
\hline $\begin{array}{l}\text { ALDH } \\
2\end{array}$ & PGR & $\begin{array}{l}\text { ADRB } \\
2\end{array}$ & CASP8 & PRKCD & $\begin{array}{l}\text { SLC2A } \\
4\end{array}$ \\
\hline $\begin{array}{l}\text { MMP1 } \\
2\end{array}$ & $\mathrm{NR} 3 \mathrm{C} 2$ & JUN & PRKCA & CCNB1 & FLT4 \\
\hline CAT & NOS2 & $\begin{array}{l}\text { CHEK } \\
1\end{array}$ & PON1 & FLT1 & $\begin{array}{l}\text { SLC2A } \\
1\end{array}$ \\
\hline $\begin{array}{l}\text { FASL } \\
\text { G }\end{array}$ & & & & & \\
\hline
\end{tabular}

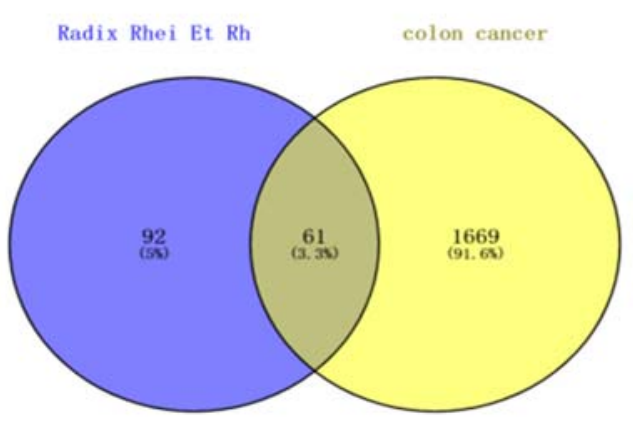

Figure 1. Venny plot of Rhubarb active ingredient target and colon cancer target

\subsection{Protein interaction networks and important targets}

STRING database (HTTPS://String-DB.org) is a database of known and data-predicted protein-protein interactions (PPI) that can be used to bring the resulting Rhubarb potential targets for colon cancer into the STRING platform. The species was set as "Homo spaiens", the confidence was set as "0.4" for screening, and the remaining parameters were kept as default. TSV file of protein interaction information of target gene was obtained, and PPI network was constructed by String database and Cytoscape software ${ }^{[5-6]}$. Cytoscape software was used to render PPI network and perform cluster analysis of important gene modules. All the default parameters in Mcode application were executed to obtain the densely connected network modules (modules) in PPI network. Targets contained in the Module were important targets of Rhubarb in the treatment of colon cancer. Figure 2 is a PPI network diagram, which contains 61 Nodes and 373 Edges. Figure 3 shows subcluster B (score 9), which contains 13 Nodes and 54 Edges, and 13 core genes are obtained, including PTGS2, IL1B, Bax, NR3C1, NOS2, CSF2, CASP3, GPT, CTSD, MYC, PPARG, JN, EGF. 


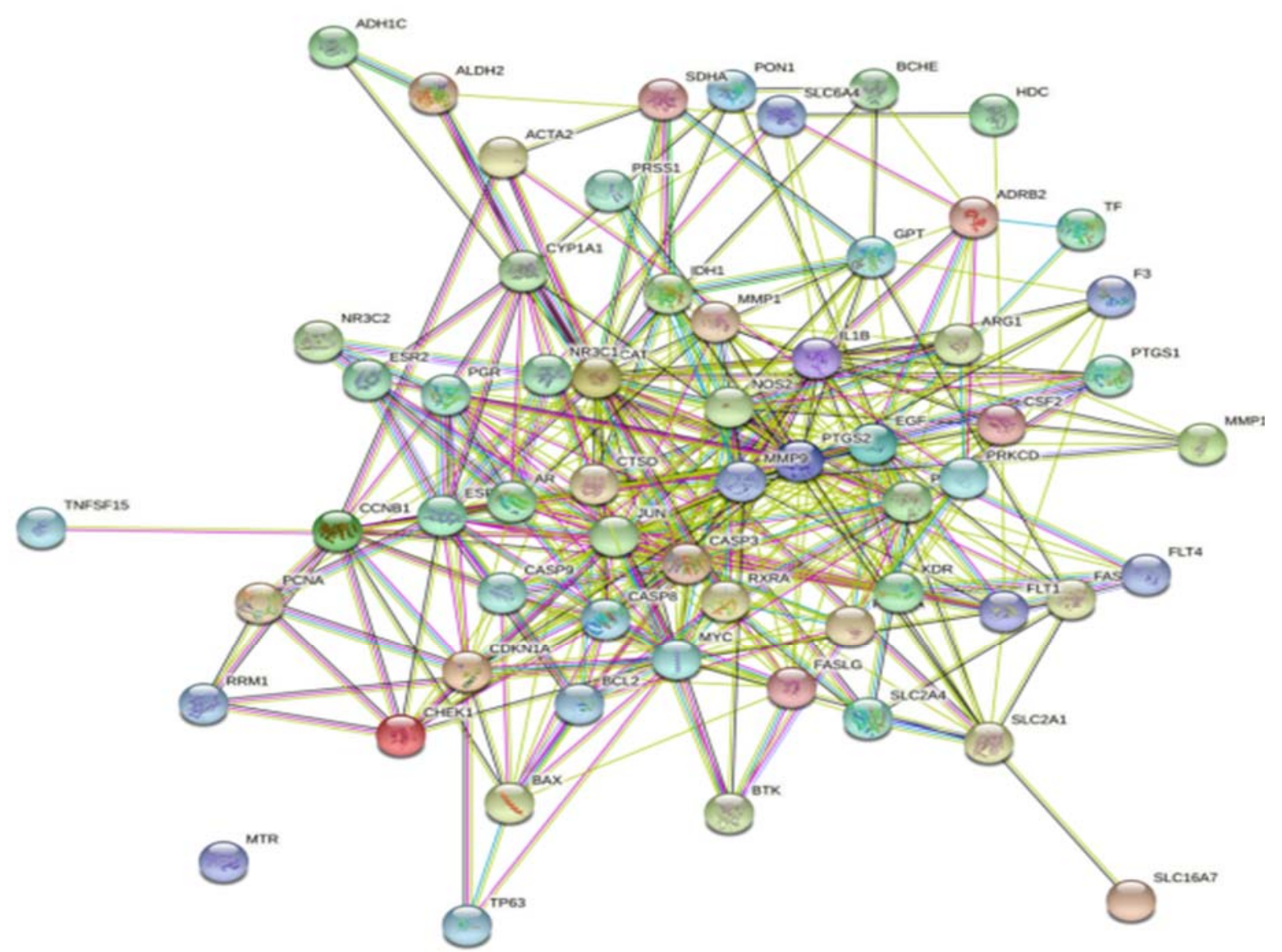

Figure 2. PPI network interaction diagram

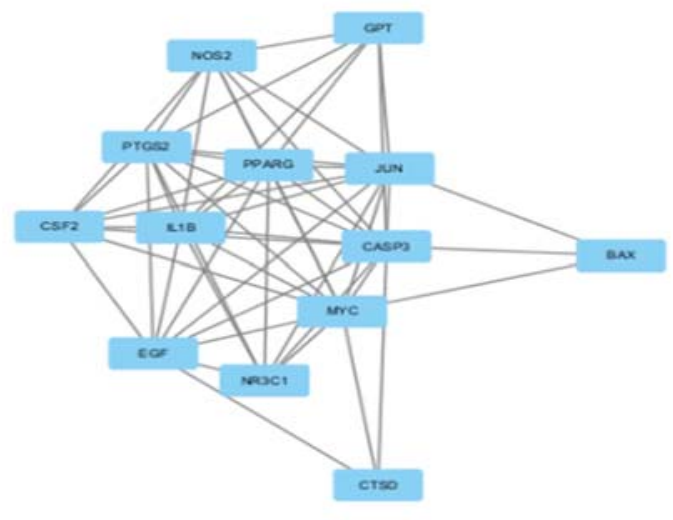

Figure 3. Subcluster B: Core gene

\subsection{Ontology analysis and KEGG pathway annotation and enrichment analysis}

Gene Ontology (GO) analysis is a general method to annotate Gene products and their functional characteristics $^{[7-8]}$. GO analysis can be divided into biological process (BP) and cellular component according to different functions (CC), Molecular Function (MF). Import the intersection target into the Metascape database (HTTPS://metascape.org), select the species type as "Homo sapiens", then select "Custom Analysis" and use the default setting as the filter condition.Go analysis and Kyoto Encyclopedia of Genes and Genomes (KEGG) pathway annotation and enrichment analysis were performed for gene annotation, and the results were visualized.

Metascape was used to carry out GO enrichment analysis and KEGG pathway enrichment analysis of 13 important targets of Rhubarb in the treatment of colon cancer. The enrichment results were sorted according to $\mathrm{P}$ value, and COUNT value represented the number of genes enriched in this pathway. GO enrichment analysis was carried out in three aspects of biological process enrichment, molecular function analysis and cell component analysis. The results are shown in Figure $4 \sim$ 7. According to the P-value and COUNT value of KEGG pathway analysis, PI3K plays a key role in the top 15 pathways related to Rhubarb in the treatment of colon cancer, as shown in Figure 4. Enrichment of GO biological processes mainly involves responses to nutrient levels, homeostasis in multicellular organisms, regulation of peptide secretion, regulation of protein secretion, and response to peptide hormones, etc., as shown in Figure 5. The molecular function analysis of GO mainly involves the molecular functions of the ciliary body, outer plasma membrane, cytoplasmic cystic cavity, cystic cavity, endoplasmic reticulum and other parts, as shown in Figure 6. The analysis of GO cell components mainly involves receptor ligand activity, cytokine receptor binding, cytokine activity, DNA-binding transcriptional activator activity, RNA polymerase specificity, hormone activity, etc., as shown in Figure 7. 


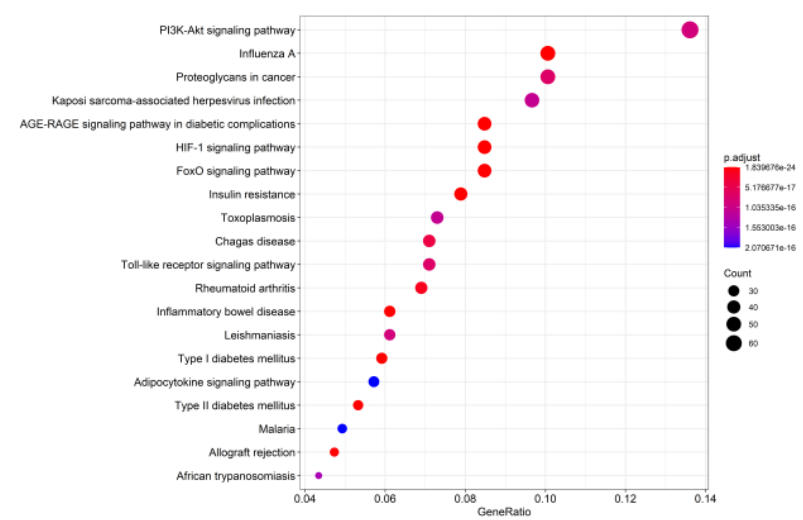

Figure 4. Bubble diagram of KEGG analysis

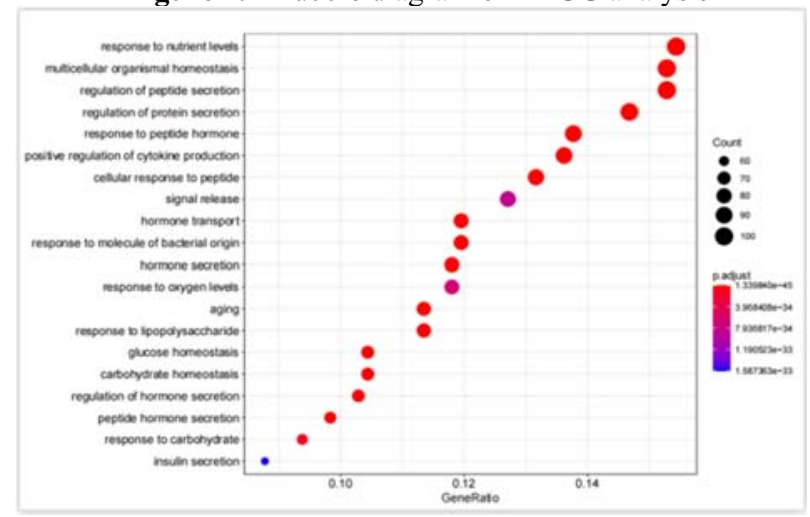

Figure 5. Bubble diagram of GO biological process

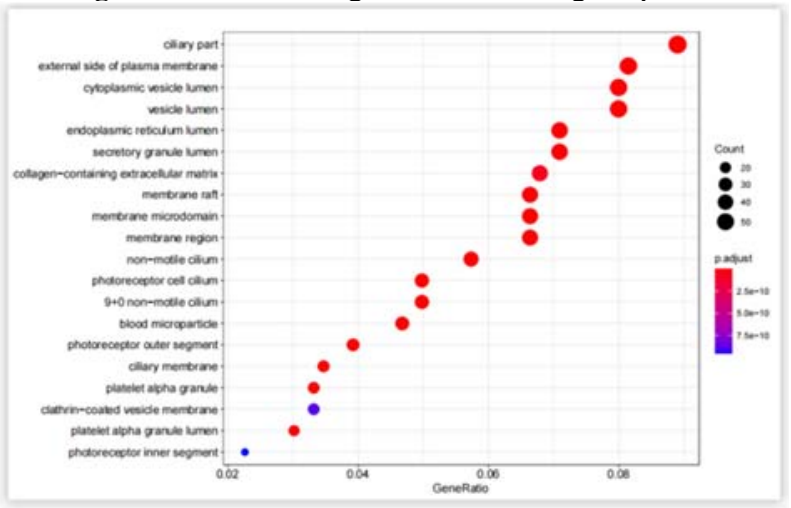

Figure 6. Bubble diagram of GO molecular function analysis

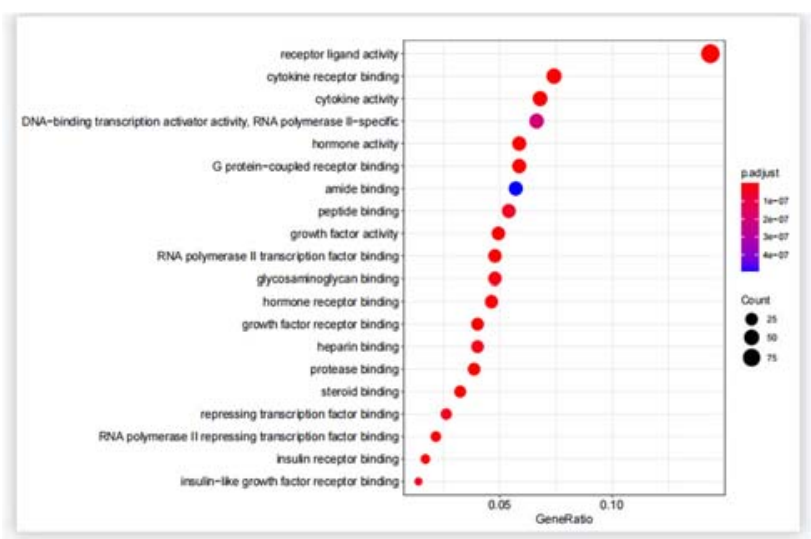

Figure 7. Bubble diagram of GO cell component analysis

\subsection{Rhubarb active ingredient - colon cancer - KEGG action network}

Cytoscape 3.7.2 software was used to construct the Rhubarb active ingredient - colon cancer - KEGG action network. In this network, Node represents the active component of Rhubarb and its target protein acting on colon cancer, while Edge represents the interaction between active component and target protein, as shown in Figure $8^{[9]}$.

\section{Conclusions}

Network pharmacology plays an important role in analyzing the pharmacological action of traditional Chinese medicine and predicting the effect of treating diseases. Through the network pharmacological study of Rhubarb in this study, it was found that Rhubarb can participate in the treatment of colon cancer through multitarget and multi-pathway.Pharmacology research through the network, KEGG pathway analysis showed that PI3K play a key role, GO enrichment of biological process shows mainly involved in response to the nutrition level, multicellular organisms, homeostasis, peptide secretion regulation, molecular function analysis showed mainly involves the ciliary body, GO cell component analysis showed that mainly involves receptor ligands.

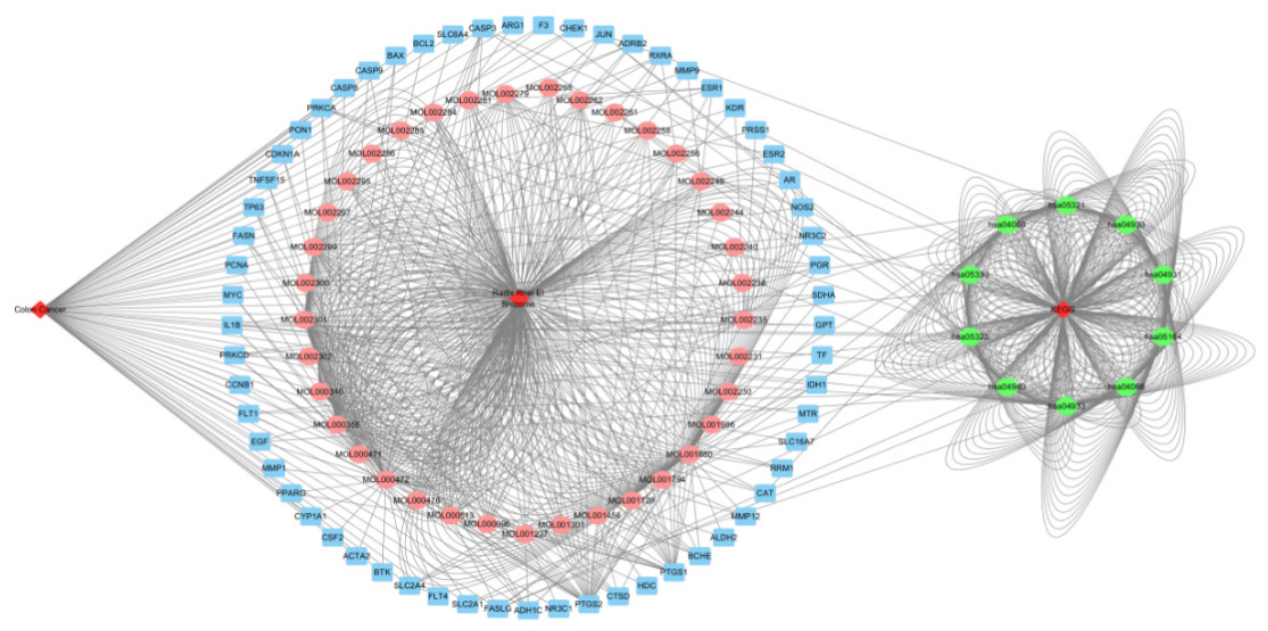

Figure 8. Rhubarb active ingredient - colon cancer-KEGG action network 


\section{References}

1. J. Ru, P. Li, J. Wang, W. Zhou, B. Li, C. Huang, P. Li, Z. Guo, W. Tao, Y. Yang, X. Xu, Y. Li, Y. Wang, L. Yang. J. cheminformatics 6, 13, (2014)

2. X. Xu, W. Zhang, C. Huang, Y. Li, H. Yu, Y. Wang. INT. J. MOL. SCI 13, 6964, (2012)

3. J. Walters. Curr Opin Gastroenterol, 2002,18(2):161167.

4. C. Zhang, L. Yang, J. Li, C. Zhong, L. Lin. Chinese a rchives of traditional Chinese medicine 38, 175, (2020)

5. Y. Luo, L. Lin, Y. Liu, G. Shi, Y. Wu, A. Yang, H. Li. China journal of Chinese materia medicine 45, 5753, (2020)

6. Y. Yang, S. He, Y. Wang, Y. Chen, C. Wang. J Dig Oncol (Electronic Version) 11, 282, (2019)

7. Y. Zong, M. Ding, K. Jia, S. Ma, W. Ju. Chinese Traditional and Herbal Drugs 51, 836, (2020)

8. Y. Deng, B. Liu, Z. He, T. Liu, R. Zheng, A. Yang, A. Huang, Y. Li. Chinese Traditional and Herbal Drugs 51, 1113, (2020)

9. H. Wu, J. Wang, Y. Yang, T. Li, Y. Cao, Y. Qu, Y. Jin, C. Zhang, Y. Sun. ACTA PHARM SIN 55, 374, (2020) 